\title{
Le quartier comme espace de résistance et de politisation
}

La Vallée de Dikmen à Ankara face à un projet de transformation urbaine The Neighborhood as a Space of Resistance and Politicization: Ankara's Dikmen Valley Faced with an Urban Transformation Project

\section{Gülçin Erdi Lelandais}

\section{(2) OpenEdition}

\section{Journals}

\section{Édition électronique}

URL : http://journals.openedition.org/conflits/19203

DOI : $10.4000 /$ conflits. 19203

ISSN : $1777-5345$

Éditeur :

CCLS - Centre d'études sur les conflits lilberté et sécurité, L'Harmattan

\section{Édition imprimée}

Date de publication : 19 mai 2016

Pagination : 139-167

ISBN : 978-2-343-09459-5

ISSN : 1157-996X

Référence électronique

Gülçin Erdi Lelandais, "Le quartier comme espace de résistance et de politisation », Cultures \& Conflits [En ligne], 101 | printemps 2016, mis en ligne le 19 mai 2017, consulté le 30 mars 2021. URL : http:// journals.openedition.org/conflits/19203; DOI : https://doi.org/10.4000/conflits.19203 


\title{
Le quartier comme espace de résistance et de politisation
}

\author{
La Vallée de Dikmen à Ankara \\ face à un projet de transformation urbaine 1
}

\section{Gülçin ERDI LELANDAIS}

Gülçin Erdi Lelandais est sociologue, chargée de recherche an CNRS et membre de I'UMR CITERES. Ses travaux portent sur les formes de solidarité et de résistance ainsi que les mobilisations urbaines. Les dimensions spatiales des représentations, des constructions identitaires et des modes d'affirmation de la citadinité dans un contexte de marginalité socio-spatiale sont au coeur de ses analyses. Elle a récemment publié Understanding the City. Henri Lefebvre and Urban Studies (sous dir.), Nerwcastle-upon-Tyne, Cambridge Scholars Publishing, 2014 ; "Émergence et résistance spatiale d'un quartier en contexte autoritaire. Le cas du quartier 1 Mayıs à Istanbul », in Marchal H., Baticle C., Regards pluriels sur l'incertain politique. Entre dérives identitaires, urbanisation, globalisation économique, réseaux numériques et féminisation du social, Paris, L'Harmattan, 2015.

$\mathrm{D}$ es projets d'aménagement et de transformation urbains sont entrepris dans l'ensemble de la Turquie depuis l'arrivée au pouvoir du Parti de la justice et du développement ${ }^{2}$ (AKP). Ils restructurent en profondeur la morphologie sociale, politique et culturelle des villes. De nombreux quartiers sont alors entièrement détruits et/ou rénovés, ce qui engendre parfois déplacements forcés, expulsions, conflits et ségrégation. Au plan national ou local, les politiques d'aménagement et d'urbanisation promues par le gouvernement relèvent moins des politiques sociales de logement que d'une logique de profit et de rente immobilière. Par ailleurs, le discours sur l'insécurité, accompagné de la volonté de constituer des espaces sécurisés, est inscrit au cœur des politiques urbaines du gouvernement et des municipalités ${ }^{3}$.

1. Je remercie Sophie Caratini pour sa lecture et ses conseils sur la version préliminaire de cet article.

2. Ce parti, issu de la transformation d'anciens partis islamistes comme Refab (Prospérité) et Fazilet (Vertu) a été fondé en 2001 et peut être qualifié de droite conservatrice, néolibérale, islamiste. Au pouvoir depuis 13 ans. Recep Tayyip Erdoğan est son ancien président ; il est actuellement dirigé par Ahmet Davutoğlu. 
Ces politiques engendrent des réactions, voire des résistances dans certains lieux ${ }^{4}$, ce qui pose la question du rapport à l'espace en tant que générateur de mémoires collectives et d'appartenances identitaires et culturelles, notamment à l'échelle du quartier. La mobilisation autour du parc Gezi en juin 2013 est un des derniers exemples emblématiques de ce type de réactions ${ }^{5}$. Comme nous le verrons dans le cas du quartier de la vallée de Dikmen, elles entraînent également une relative politisation des habitants dans certains quartiers ${ }^{6}$ ou arrondissements qui n'étaient pas particulièrement engagés dans des activités militantes avant la mise en œuvre de ces projets. Plusieurs quartiers, jusqu'alors méconnus, réputés paisibles, et parfois même acquis à la majorité gouvernementale, ont ainsi commencé à se mobiliser dès 2004 contre les politiques de transformation urbaine initiées à la même période par le gouvernement ${ }^{7}$.

L'urbanisation et les politiques de logement à l'œuvre en Turquie depuis le début des années 2000 présentent des caractéristiques qui incitent parfois les chercheurs à les qualifier de néolibérales ${ }^{8}$. De nombreux travaux, notamment

3. Işın E. F., «The Neurotic Citizen", Citizenship Studies, vol. 8, n 3, 2004, pp. 217-235.

4. De nombreux travaux, notamment anglophones, ont été réalisés ces dernières années pour analyser les différentes formes de contestation émergentes face aux grands projets d'aménagement urbain en Turquie. Parmi ces travaux, on peut citer Petit C., "Engagement militant et politisation des mobilisations au sein des oppositions urbaines à Istanbul ", EchoGéo [En ligne], $\mathrm{n}^{\circ}$ 16, 2011, mis en ligne le 4 juillet 2011, consulté le 3 octobre 2012. URL : http://echogeo.revues.org/12445 ; Özdemir E., Contesting neoliberal urbanisation: contemporary urban movements in istanbul, the case of Gülsuyn-Gülensu Neighbourboods, thèse de doctorat en aménagement urbain et régional, Ankara, Université technique du MoyenOrient, 2013 ; Karaman O., "Resisting urban renewal in Istanbul », Urban Geography, vol. $35, n^{\circ} 2,2014$, pp. 290-310.

5. Cette mobilisation sociale a émergé suite à l'abattage des arbres dans le parc public de Gezi situé au niveau de la place Taksim, prélude à la destruction totale du parc dans le cadre d'un projet d'aménagement urbain. Elle a par la suite évolué vers une mobilisation politique contre le gouvernement. Pour une information détaillée sur cette mobilisation, voir Erdi Lelandais G., "Gezi protests and beyond: Urban resistance in the context of neoliberal urbanism in Turkey ", in Thörn H., Thörn C., Sernhede O., Mayer M. (dir.), Understanding Urban Uprisings, Protests and Movements: European Cities and the Crisis of Neoliberalism, Londres, Palgrave, 2016 (à paraître), pp. 283-309.

6. Nous employons la notion de « quartier» dans le sens du lieu de voisinage et de socialisation. La traduction du quartier en turc est « mahalle » qui est souvent utilisé pour parler du lieu de voisinage par les habitants. Dans ce sens, ce que nous appelons « la Vallée de Dikmen » renvoie au mahalle des habitants.

7. Une des plus emblématiques de ces luttes émergeant au sein des quartiers dits « apolitiques » fut celle du quartier Basibüyük situé dans l'arrondissement de Maltepe sur la partie asiatique d'Istanbul. Voir Sen B., Türkmen H., "Bir Kentsel Dönüşüm Sınaması: Başıbüyük Mahallesi » (Une tentative de transformation urbaine : quartier de Basibüyük) in Türkün A. (dir.), Mülk, Mahal, Insan: İstanbul'da Kentsel Dönüşüm Istanbul, Bilgi Ün. Yay, 2014, pp. 143-188.

8. On peut notamment voir Batuman B., « Minarets without Mosques: Limits to the Urban Politics of Neo-liberal Islamism », Urban Studies, vol. 50, n 6, 2013, pp. 1097-1113; Enlil Z. M., «The Neoliberal Agenda and the Changing Urban Form of İstanbul », International Planning Studies, vol. 16, n 1, 2011, pp. 5-25 ; Lovering J., Türkmen H., «Bulldozer Neoliberalism in Istanbul: The State-led Construction of Property Markets, and the Displacement of the Urban Poor ", International Planning Studies, vol. 16, n 1, 2011, pp. 7396. 
anglo-saxons 9, mettent en exergue une néolibéralisation des politiques urbaines depuis les années 2000, phénomène qui se traduit, selon Marianne Morange et Sylvie Fol, par une « adhésion des villes à l'entrepreneuriat urbain afin de développer leur attractivité pour faire face à la compétition internationale entre elles. Elle s'accompagne d'un renoncement à la planification spatiale au profit du nouveau régionalisme compétitif incarné par la planification urbaine stratégique $10 »$. Les politiques sociales de la ville et la recherche d'une certaine justice spatiale favorisant la mixité sociale et l'accompagnement des populations défavorisées sont progressivement reléguées au second plan ${ }^{11}$.

Il ne s'agit pas ici de mener une discussion approfondie sur l'influence du néolibéralisme dans la conception des villes ni sur l'émergence des résistances ${ }^{12}$. Néanmoins, on peut avancer l'idée qu'il existe une corrélation, en Turquie, entre la montée des revendications urbaines, notamment sous la bannière du «droit à la ville 13 ", et la néolibéralisation de l'espace urbain ${ }^{14}$. Ce lien est constamment souligné, tant par des organisations politiques que par les habitants luttant contre les projets d'urbanisation. Il arrive, comme nous le verrons, que ces résistances entraînent une forme de politisation conduisant à une remise en cause des fondements de ce système économique, mais aussi du régime politique turc.

Cet article a pour but d'étudier les pratiques de résistance et les mécanismes de politisation à l'œuvre en réaction à un projet de transformation urbaine en cours de réalisation dans un quartier nommé « la vallée de Dikmen », situé dans l'arrondissement de Çankaya à $5 \mathrm{~km}$ du centre-ville d'Ankara ( $c f$. la carte du découpage administratif).

Dans une première partie, nous présenterons le quartier de la vallée de Dikmen ${ }^{15}$, la place qu'il occupe dans le système urbain, ainsi que les nouvelles

9. Parmi les plus influents de ces travaux, on peut citer ceux de Brenner N., «Globalization as reterritorialization: the re-scaling of urban governance in the European Union ", Urban Studies, vol. 36, n 3, 1999, pp. 431-451 ; Harvey D., A Brief History of Neoliberalism, Oxford, Oxford University Press, 2005 ; Peck J., Tickell A., « Neoliberalizing space », Antipode, vol. 34, n 3, 2002, pp. 380-404 ; Swyngedouw E., Moulaert F., Rodriguez A., "Neoliberal Urbanization in Europe: Large-Scale Urban Development Projects and the New Urban Policy ", Antipode, vol. 34, n 3, 2002, pp. 542-577.

10. Morange M., Fol S., «Villes, néolibéralisation, justice », Justice spatiale [en ligne], $n^{\circ}$ 6, juin 2014, consulté le 5 janvier 2016. URL : www.jssj.org/article/neoliberalisation-ville-et-justicespatiale/

11. Merklen D., Quartiers populaires, quartiers politiques, Paris, La Dispute, 2009.

12. Pour une discussion théorique sur ce sujet au sein de la science politique française, voir Béal V., «Does neoliberalisation matter? Apports et limites d'une notion montante des urban studies dans la science politique française », Working papers du programme Villes \& territoires, 2010/1, Paris, Sciences Po.

13. Lefebvre H., Le Droit à la ville, Paris, Economica, 1968, 3e édition.

14. Künkel J., Mayer M. (dir.), Neoliberal Urbanism and its Contestations - Crossing Theoretical Boundaries, Basingstoke/New York, Palgrave Macmillan, 2012.

15. Nous avons réalisé l'étude du terrain sur ce quartier en octobre 2013 et en janvier 2015. Nous avons opté pour l'étude d'un quartier à Ankara, la ville capitale de la Turquie mais pourtant 
orientations des politiques d'urbanisation d'Ankara. Nous analyserons, ensuite, l'émergence de la contestation et le rôle du quartier comme espaceenjeu dans la résistance et la politisation des habitants face au projet de transformation urbaine concernant leur quartier. Que peut-on dire de la genèse de cette politisation ? Par quels outils et mécanismes les habitants se mobilisent et se politisent-ils ? Nous étudierons, enfin, les acteurs de la politisation et les différentes stratégies de résistance à la fois individuelles et collectives qu'ils mettent en œuvre.

\section{Le quartier de la vallée de Dikmen et le processus d'urbanisation à Ankara}

À partir des années 1950, l'exode rural s'impose comme la pierre angulaire de la mise en ouvre des plans d'urbanisation en Turquie. Ce processus commence, dès les années 1930, à Ankara, capitale de la nouvelle République 16, et entraîne l'apparition des gecekondus 17 dans la ville. Face à cette migration intensive, dans une période où l'offre privée de logement est limitée, les institutions publiques ne sont pas en mesure de proposer suffisamment de logements sociaux ou des solutions alternatives d'hébergement pour les nouveaux arrivants. Charles Hart résume ce constat de la manière suivante :

«Partout dans le monde, les paysans qui migrent vers les villes rencontrent plus de difficultés à trouver un logement qu'à trouver un emploi... Les Turcs ont résolu ce problème par les gecekondus. Le gouvernement ayant été incapable de construire un nombre suffisant de logements, et les entrepreneurs n'ayant pas trouvé rentable de s'investir dans la construction de ce type de logement populaire,

très peu étudiée par la sociologie urbaine. L'ensemble des travaux sur la ville en Turquie se concentre sur Istanbul en rendant, de fait, invisibles de nombreux conflits, mobilisations et résistances dans le reste du pays. Notre objectif était donc de rendre visible ce qui est invisible et d'apporter une contribution pour nuancer les constats faits uniquement à partir du cas d'Istanbul. Dans ce cadre, nous avons choisi une enquête qualitative avec des entretiens semidirectifs individuels et en groupe. Nous avons également participé aux réunions du Bureau du droit au logement. Nous avons effectué des visites chez les habitants et avons observé leurs pratiques quotidiennes dans leur cadre de vie. 20 personnes du quartier et 6 personnes des organisations militantes œuvrant pour une urbanisation alternative (Chambre des ingénieurs et des architectes, Halkevleri, chercheurs de l'Université technique du Moyen-Orient (ODTÜ) et de l'Université d'Atılım) ont été interviewées. Je remercie particulièrement Mert Anıl Eren et Bilge Hasdoğan, deux doctorants de l'ODTÜ, dont l'aide sur le terrain fût précieuse.

16. Le travail le plus détaillé et le plus complet sur l'urbanisation d'Ankara au XXe siècle a été réalisé par Tans1 Şenyapılı qui constate, dès les années 1930, l'installation des migrants d'Anatolie sur des terrains publics et la construction de baraques sans autorisation. Voir Şenyapılı T., 'Baraka'dan Gecekonduya. Ankara'da Kentsel Mekanın Dönüşümü 1923-1960 [De la baraque au gecekondu. La transformation de l'espace urbain à Ankara 1923-1960], Istanbul, İletişim, 2004.

17. Mot utilisé pour désigner l'habitat informel en Turquie. Il signifie littéralement «maisons construites en une nuit ». Pour l'approfondissement théorique, voir le numéro spécial "Gecekondu », in European Journal of Turkish Studies, n 1, 2004 qui propose une réflexion sur l'usage et les définitions du terme " gecekondu » qui est souvent utilisé sans être conceptualisé dans les études urbaines turques. Nous n'approfondirons pas davantage le débat sur le «gecekondu» qui n'est pas l'objet de cet article. 
les migrants ruraux se sont mis à construire clandestinement leurs maisons de préférence sur les propriétés foncières non-occupées de l'État... 18 ».

Par conséquent, les migrants construisent spontanément leur quartier d'habitation par leurs propres moyens. Dès les années 1950, on constate la multiplication et une large dissémination de ces habitats précaires dans la ville, et pas seulement à sa périphérie, ce qui engendre une expansion démographique spectaculaire ( $c f$. tableaux 1 et 2). Pendant la période 1960-1980, ces zones de gecekondu deviennent progressivement des espaces communs où des relations de réciprocité, d'échange et de solidarité se développent parmi les migrants qui tendent à s'y installer dans une logique de proximité ethnique, géographique et même parfois religieuse et politique ${ }^{19}$.

Tableau 1 : Progression de la population à Ankara 20

\begin{tabular}{lccccccc}
\hline Année & 1831 & 1990 & 1927 & 1955 & 1965 & 1980 & 2014 \\
\hline Population & 22000 & 35051 & 74553 & 451241 & 905660 & 2561761 & 5150072 \\
\hline
\end{tabular}

Tableau 2 : Construction des gecekondus à Ankara

\begin{tabular}{lccccc}
\hline Période & $1915-49$ & $1950-59$ & $1960-69$ & $1970-79$ & $1980-89$ \\
\hline Pourcentage & 1.5 & 4.5 & 16.9 & 58.7 & 18.4 \\
\hline
\end{tabular}

Source : Balaban U., "The Enclosure of Urban Space and Consolidation of the Capitalist Land Regime in Turkish Cities », Urban Studies, vol. 48, n 10, 2011, pp. 2162-2179.

À l'époque de la fondation de la ville, la vallée de Dikmen était considérée comme l'un des «couloirs d'aération » d'Ankara, située pour sa part dans une cuvette entourée de montagnes. Initialement, ce qu'on appelle aujourd'hui Dikmen était un petit village avec quelques jardins-vignobles, relativement excentré de la nouvelle ville conçue en 1928 selon les plans d'urbanisation d'Herman Jansen. Ces plans avaient comme perspective une ville comptant au maximum 300000 habitants à l'horizon des années 1980. Ils prévoyaient donc de préserver le village de Dikmen et ses environs, afin de maintenir ses activités agricoles, d'assurer la qualité de l'air et de conserver des espaces verts ${ }^{21}$.

18. Cité dans Balaban U., «The Enclosure of Urban Space and Consolidation of the Capitalist Land Regime in Turkish Cities ", Urban Studies, vol. 48, n 10, 2011, p. 2164.

19. Karpat, K. H., The Gecekondu: Rural Migration and Urbanization, Cambridge, Cambridge University Press, 1976 ; Tok E., A la Turka Neoliberalism. Gecekondus as Turkey's New State Spaces, Saarbrücken, Lambert Academic Publishing, 2010.

20. Ce tableau a été constitué à partir des tableaux cités en annexe de l'ouvrage de Tansı Şenyapılı susmentionné ainsi que des données de l'Institut national des statistiques de Turquie (TÜíK) : http://rapory.tuik.gov.tr/11-05-2015-17:47:04-14661238601079071629313302904.html

21. Şenyapili T., op. cit., pp. 63-73. 
La forte pression démographique inattendue a rendu caducs les plans de Jansen. Le besoin urgent et massif de logements pour les nouveaux arrivants a élargi progressivement les limites de la ville, et la vallée de Dikmen est une des zones où les gecekondus se sont multipliés dès les années 1960. La construction de ces habitations s'est accélérée dans les années 1970 dans certains quartiers comme Dikmen et Mamak Tuzluçayır sous l'impulsion de divers groupes radicaux de gauche. Ces organisations, plus ou moins informelles, cherchaient alors des terrains inoccupés dans différents quartiers ankariotes afin de les distribuer - parfois en usant de la force - aux immigrés ouvriers. Un des habitants et fondateurs d'un quartier gecekondu relate ce processus :

«Pendant mes années de jeunesse, quand je travaillais à l'université, l'exode rural était massif. Dans les conditions politiques de l'époque, nous, en tant que socialistes essayant de faire quelque chose pour le peuple, réfléchissions sur des solutions pour satisfaire les besoins de logement des nouveaux arrivants dans la ville. Nous avons repéré des terrains publics à Piyangotepe, Yenimahalle et Dikmen susceptibles d'être distribués à ces derniers pour qu'ils puissent construire ensemble des maisons. Au départ, les villageois n'ont pas voulu donner leur terre ici à Dikmen. Nous n'avons pas réussi à les convaincre et avons même eu des affrontements. À la fin, nous avons accaparé les terres et nous les avons distribuées par la suite 22 ».

L'intérêt porté à ces zones d'habitat est, avant tout, fondé sur la conviction des organisations révolutionnaires estimant qu'il faut considérer les gecekondus comme des unités de mobilisation à l'instar des usines. Les gecekondus sont, à n'en pas douter, des lieux d'accueil de la pauvreté et de la misère. Ils constituent par conséquent un terreau fertile pour la mobilisation et la formation active d'une conscience de classe. Autrement dit, pour les organisations de l'époque, le peuple des gecekondus était plus susceptible qu'un autre de passer d'une «classe en soi » à une « classe pour soi » selon la terminologie marxiste. De nombreux groupes socialistes composés de jeunes et rassemblés autour de revues politiques - dont les plus connues sont Halkin Yolu (Chemin du peuple), Devrimci Yol (Sentier révolutionnaire) et Partizan vont donc accorder une importance particulière aux habitants des gecekondus et se montrer solidaires envers eux ${ }^{23}$.

22. Entretien avec Tarık Çalışkan, Ankara, 29 octobre 2013.

23. Pour une discussion détaillée sur le rôle des groupes de la gauche radicale dans la construction des quartiers de gecekondu, voir Sen B., "The Production of Autonomous Settlements for the Working Class by the Turkish Socialist Movements ", papier présenté au colloque Resourceful Cities dans la Session 26 : Autonomous urban movements: socio-spatial structures and political impacts, Berlin, 2013 ; Lelandais G., "Émergence et résistance spatiale d'un quartier en contexte autoritaire. Le cas du quartier 1 Mayıs à Istanbul », dans Marchal H., Baticle C. (dir), Regards pluriels sur l'incertain politique. Entre dérives identitaires, urbanisation, globalisation économique, réseaux numériques et féminisation du social, Paris, L'Harmattan, 2015, pp. 109-133. 


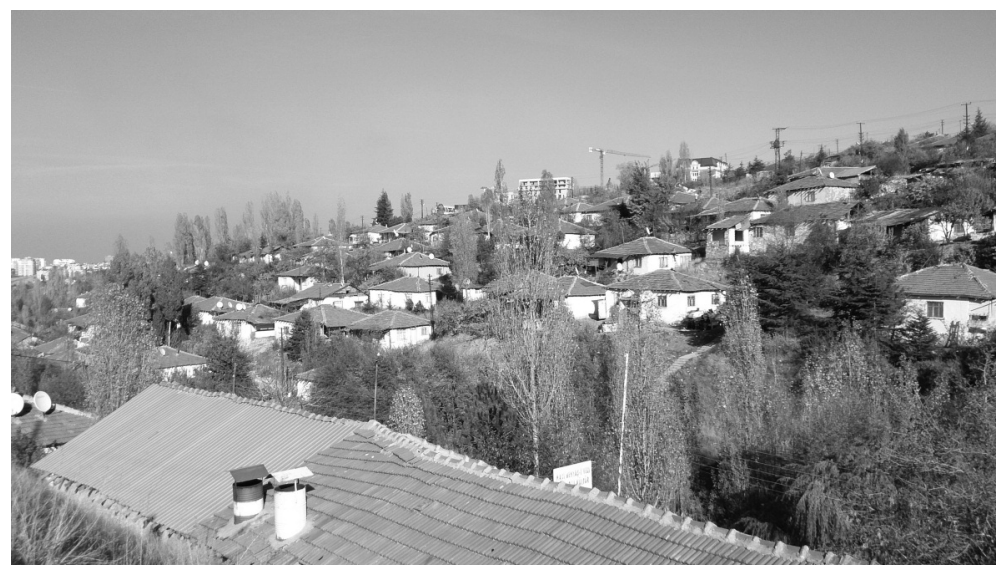

Gecekondus dans la Vallée (cliché de l'auteure)

À sa création, le quartier de la vallée de Dikmen n'est quasiment pas relié au centre-ville d'Ankara et il est difficile d'accès. En outre, il ne dispose d'aucune infrastructure ou service de base tels que routes, réseaux d'électricité ou eau, etc. D'ailleurs, les raccordements à ces deux derniers services s'effectuent de manière clandestine. En raison de l'augmentation rapide de la population et des gecekondus, les décideurs politiques sont finalement contraints de reconnaître administrativement ce quartier et d'y implanter des équipements publics élémentaires. Une partie des habitants parvient même à obtenir de la municipalité des certificats d'attribution de propriété dans le courant des années 1980. Si ces décisions s'imposent au vu de la taille du quartier et de la population, elles répondent également largement à des considérations électorales : avec la légalisation du bâti et la reconnaissance du quartier, les différents gouvernements successifs espèrent obtenir des voix.

En 1989, la municipalité métropolitaine d'Ankara et la municipalité de Çankaya, toutes deux dirigées par le Parti républicain du peuple (CHP) ${ }^{24}$, décident de procéder à une rénovation urbaine et inscrivent le « Projet de la vallée Dikmen » dans les plans directeurs ${ }^{25}$ d'Ankara. Une société semipublique, Metropol İmar AŞ, est créée en partenariat avec la municipalité de Çankaya pour entreprendre la première étape de ce projet qui en comporte six correspondant à des blocs de construction successives tout au long de la vallée. De nombreuses enquêtes sont menées auprès des habitants, des cartes de la zone à diverses échelles et des études géologiques sont réalisées ${ }^{26}$. Les plans

24. Un parti de centre-gauche, kémaliste, social-libéral, progressiste. Historiquement fondé par Mustafa Kemal Atatürk, le CHP présente un caractère social-démocrate et étatiste dans les années 1990, pour ensuite basculer dans une approche ultra-nationaliste entre 2000 et 2010 notamment. Le parti s'est engagé depuis quelques années dans une voie progressiste, davantage pluraliste et plutôt non-offensif avec le mouvement kurde. Il est dirigé par Kemal Kilıçdaroğlu.

25. Un plan directeur détermine les orientations globales des politiques d'urbanisation d'une ville. 
d'aménagement prévus par ce projet poursuivent trois grands objectifs :

- Créer un couloir de circulation de l'air avec une ceinture verte et réduire, de ce fait, la pollution de l'air.

- Développer une landmark avec des zones culturelles et récréatives.

- Fournir des logements à bas coût aux habitants ${ }^{27}$.

Au total, plus de 4092 appartements sont construits par Metropol İmar. Sur ce nombre, ce dernier en a récupéré 2046 ; le reste, quant à lui, est distribué aux familles possédant un gecekondu dans la zone de la première étape du projet 28 . Les objectifs ne sont pas tous remplis, mais les premières étapes du projet sont réalisées avec une relative consultation des habitants et la prise en considération de leur droit au relogement. Après l'arrivée de Melih Gökçek (il est d'abord membre du parti de la mère-patrie, ANAP de centre-droit, puis passe à l'AKP) à la tête de la municipalité métropolitaine d'Ankara en 1994, les caractéristiques et l'esprit du projet sont modifiées. Désormais, l'objectif poursuivi est davantage la maximisation du profit foncier que la création de logements et la promotion de projets sociaux ${ }^{29}$.

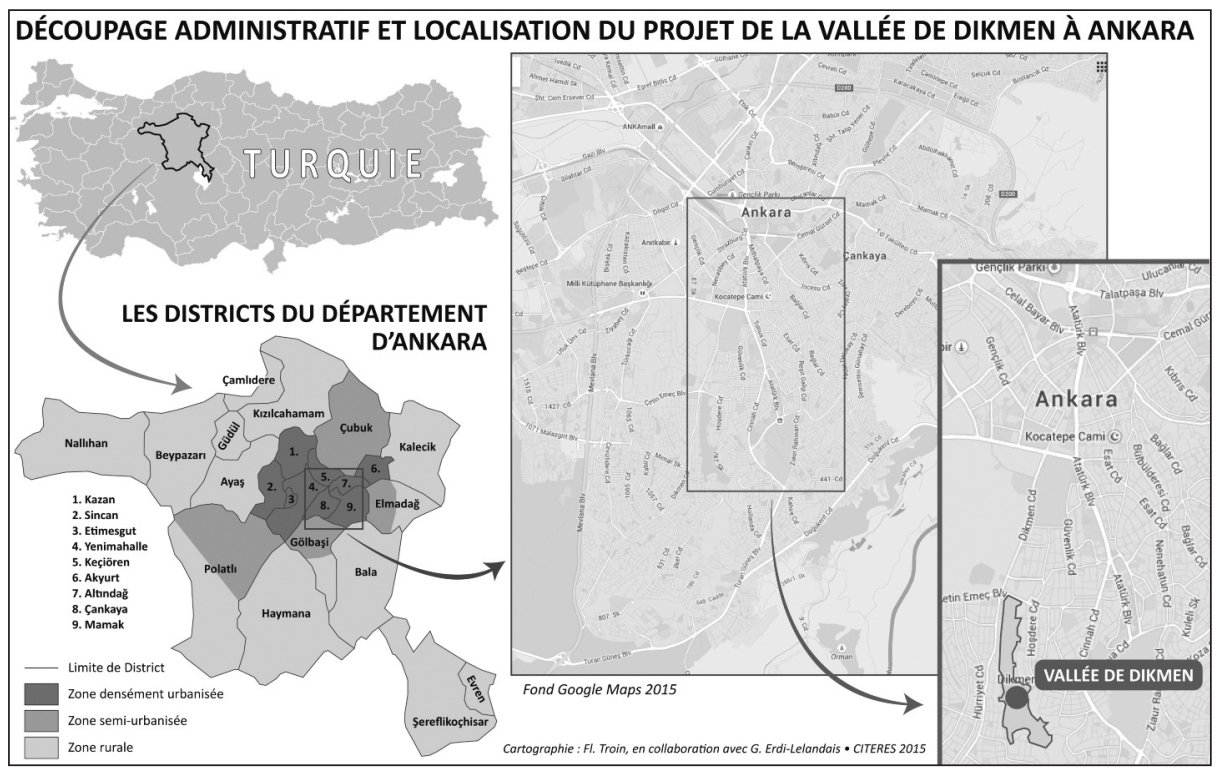

26. Entretien réalisé avec Zafer, un ancien fonctionnaire de la mairie à l'époque de la mise en place du projet de Dikmen, Ankara, 13 janvier 2015.

27. Akyürek S., «The Dikmen Valley Project and Its Evaluation », Study Paper, Ankara, Middle East Technical University (METU), 1993.

28. La distribution a été faite selon le principe d'un gecekondu = un appartement dans les immeubles nouvellement construits. Voir Malusardi F., Occhipinti G., "Informal Settlements Upgrading: the Gecekondus in Ankara », 39th ISoCaRP Congress, Le Caire, 2003.

29. Akça I., Bekmen A., Özden B.A., Turkey Reframed. Constituting Neoliberal Hegemony, Londres, Pluto Press, 2014. 
Depuis 2003, avec le renouvellement du statut de la Direction des logements collectifs (Başbakanlık Toplu Konut İdaresi Başkanliğ , TOKİ), le gouvernement de l'AKP accorde une importance particulière aux projets d'aménagement public, que ce soit en matière de transformation urbaine ou de projets de développement de grande ampleur comme la construction des barrages ou des autoroutes. La construction est considérée comme un secteur-clé dont la vitalité est indispensable au maintien de la croissance et à la stabilité de l'économie turque. L'objectif est alors de mener sans répit des projets liés à ce secteur pour créer de nouvelles ressources économiques et alimenter autant que possible la croissance économique turque ${ }^{30}$.

Contrairement aux années 1980 et 1990, décennies durant lesquelles les projets de rénovation étaient entrepris dans des zones périphériques, il s'agit désormais de restructurer au cœur des villes des zones considérées comme insalubres, mais à fort potentiel immobilier, pour y installer des populations socialement et financièrement aisées. Conformément à une tendance globale, ce phénomène que l'on peut qualifier de gentrification commence à dominer la conception actuelle des métropoles en Turquie. En général, ces nouveaux aménagements sont réalisés sans véritable processus de concertation et de négociation avec les habitants des quartiers visés, à savoir les classes populaires. Celles-ci sont, la plupart du temps, victimes d'expropriations forcées et ne se voient proposer qu'un seul type d'habitat de substitution, situé à la périphérie de la ville.

Cette évolution affecte également le projet de la vallée de Dikmen. En 2006, la municipalité métropolitaine d'Ankara déclare le lancement des étapes quatre et cinq du projet dans des conditions qui restreignent les modes d'accès à la propriété des habitants de gecekondu. Contrairement aux étapes précédentes, non seulement la possession d'un gecekondu ne permet plus à ses occupants d'obtenir un appartement sur place et sans endettement supplémentaire, mais ils sont de surcroît contraints, s'ils veulent être relogés, à s'installer dans une zone périphérique (le lieu-dit Mamak Kusunlar) d'Ankara dépourvu de véritables infrastructures ${ }^{31}$. En juillet 2006, le maire d'Ankara annonce sur plusieurs antennes télévisées locales la mise en œuvre imminente de ces étapes du projet, affirmant que les habitants de la vallée de Dikmen ont profité gratuitement de ce lieu depuis des années et qu'ils doivent maintenant signer un accord avec la mairie sous quinze jours, faute de quoi leur maison sera entièrement détruite, sans aucune indemnisation ni aucun droit de relogement. Il qualifie également les habitants de çapulcu ${ }^{32}$ et prévient que la mairie

30. Çavuşoğlu E., Strutz J., "'We'll Come and Demolish Your House!': The Role of Spatial (Re)Production in the Neoliberal Hegemonic Politics of Turkey ", in Akça I., Bekmen A., Özden B. A. (dir.), Turkey Reframed. Constituting Neoliberal Hegemony, op. cit., pp. 141153.

31. Özer M. (dir.), Orada Hayat Var [Il y a une vie là-bas], Ankara, TMMOB, 2012, pp. 28-29.

32. On peut traduire ce mot par maraudeur ou pilleur. Il est couramment employé en Turquie, 
ne fera preuve d'aucune tolérance envers ceux qui s'obstinent à refuser de quitter le quartier. Ces menaces et ces insultes auront par la suite un effet mobilisateur pour les habitants de la vallée.

\section{L'émergence de la résistance et le quartier comme lieu de politisation}

La mobilisation et la politisation du quartier de la vallée de Dikmen sont donc largement liées à la mise en œuvre de la dernière étape du projet d'aménagement urbain. Au départ partiellement inclusif et « non lucratif 33 » selon la mairie social-démocrate ainsi que relativement attentif aux conditions sociales et aux sensibilités des habitants, ce projet de transformation revêt peu à peu une dimension lucrative et semble privilégier, pendant le mandat de Melih Gökçek, l'augmentation de la rente foncière ${ }^{34}$ dans cette partie de la ville. Les habitants voient ainsi leur droit d'accès aux nouveaux logements sur place négligé et leurs indemnités limitées par les décideurs publics. Nombre d'entre eux sont en effet obligés de quitter leur maison et d'accepter les solutions de relogement proposées. Considérée comme une injustice par une partie des habitants, cette situation ouvre la voie à l'organisation d'une résistance. Émerge alors, à l'automne 2006, une mobilisation qui se poursuit aujourd'hui sous diverses formes et à une autre échelle, puisque le débat est désormais porté au niveau national, voire international.

L'organisation de la résistance naît au sein des habitants qui refusent l'accord imposé par la mairie. Mais elle ne se déroule pas sans conflit, et ne concerne pas tout le monde. Dès le départ, de nombreux foyers ont accepté le projet : ils ont signé des contrats avec la mairie et accepté les conditions proposées et la construction de leur nouveau logement. Compte tenu de notre problématique, nous avons fait le choix de focaliser ici notre propos sur ceux qui ont décidé de se mobiliser.

Bien que des différences ethniques (Turc-Kurde), religieuses (AléviSunnite) et politiques (Gauche, Extrême-Gauche, Nationalistes, Conservateurs) dispersent et divisent quelque peu les habitants contestataires et compliquent initialement l'organisation de la mobilisation, certains d'entre eux - dont quelques fondateurs du quartier au passé militant - parviennent à se faire entendre et proposent des réunions. Au début, la plupart des habitants se méfient de ces personnages qu'ils croient Alévis ${ }^{35}$. En raison de leur rébel-

notamment après la résistance du parc Gezi, pour qualifier toute sorte de protestataire se rebellant contre l'État.

33. Selon les dires d'un ancien fonctionnaire de la mairie de l'époque, Zafer, responsable du projet pendant les première et deuxième étapes.

34. Pour une discussion approfondie sur le rôle de la rente foncière dans l'urbanisation actuelle, voir Swyngedouw E., Moulaert F., Rodriguez A., «L’urbanisation néolibérale en Europe : grands projets urbains et nouvelle politique de la ville », in Gintrac C., Giroud M. (dir.), Villes contestées. Pour une géographie critique de l'urbain, Paris, Les prairies ordinaires, 2014, pp. 143-162. 
lion dans les années 1930, de leur engagement à gauche dans les années 1970, puis de la répression qui s'en est suivie, les Alévis sont souvent perçus comme l'ennemi intérieur en Turquie ${ }^{36}$. De ce fait, ils adoptent toujours une posture critique vis-à-vis de l'État et se positionnent majoritairement à gauche. De par cet héritage militant historique, ils ont acquis une culture de la résistance et une primo-socialisation politique qui sert depuis lors de base à tout type de mobilisation et d'action collective. Leur passé radical et notamment l'apparence physique de l'un d'entre eux faisant penser qu'il est Alévi (en raison de sa longue barbe) n'incitent pas certains à participer aux réunions d'informations qu'ils organisent dans le quartier:

"Quand je suis venu ici, il y avait des gens qui me connaissaient et
d'autres non. Ils m'appréciaient à partir de mon apparence phy-
sique. Ils disaient “ce type ne ressemble à rien... c'est un gars com-
muniste, il ne faut pas le suivre". Par exemple, un des habitants avait
détruit sa maison lui-même en disant qu'il ne me suivrait pas
puisque je suis Alévi et donc douteux. Il a su par la suite que je
n'étais pas Alévi et a dit aux autres qu'il allait s'excuser [rires]. Or,
moi j’avais juste décidé de laisser pousser ma barbe jusqu'au jour où
Gökçek [maire] accepterait nos revendications. C'était juste un
pari 37 ».

Après plusieurs réunions, les habitants sont finalement convaincus de la sincérité de ces personnes et de la nécessité de la lutte; ils décident ensuite d'élire des « représentants de rue », membres de l'assemblée du quartier. Le principe de ce fonctionnement est que, pour chaque rue du quartier, il faut nommer un responsable qui sera chargé d'informer les habitants sur l'avancement du projet et sur les actions à mener. Ces représentants de rue sont alors choisis au cours des réunions et en fonction du degré d'intensité de leur implication dans la contestation du projet, il ne s'agit donc pas forcément de militants confirmés de longue date. Enfin, lorsqu'ils sont tous nommés, ils s'accordent entre eux sur le lieu dans lequel ils se rassembleront régulièrement pour conduire la mobilisation. Pratiquement au même moment, la municipalité installe en haut de la vallée un «bureau des démolitions » pour diriger la mise en œuvre du projet et instituer les relations administratives avec les habitants. En réaction, l'assemblée des représentants de rue crée le «bureau du droit au logement » et l'installe également dans un gecekondu, avec des équipements (ordinateur, tables, chaises, imprimante, radiateur, etc.) réunis grâce

35. Les Alévis ont en général des pratiques séculières et sont très attachés à la République. Leurs pratiques religieuses se différencient clairement du sunnisme et excluent les représentations publiques de la religion. Ils ont leur propre lieu de culte, Cemevi, à la place de la mosquée.

36. Massicard É., «La judiciarisation contrastée de la question alévie. De la Turquie à l’Europe », Revue française de science politique, vol. 64, ${ }^{\circ} 4,2014$, pp. 711-733.

37. Extrait d'entretien avec Tarık Çalışkan, Ankara, 29/10/2013. Pour voir le personnage : http://sendika8.org/2012/10/tarik-caliskanin-evi-kursunlandi/; http://tetesdeturques.tumblr.com/ 
à l'entraide des habitants qui participent à la mobilisation ${ }^{38}$. Détruit une première fois, puis brûlé par des hommes de la mairie, selon les dires des habitants ${ }^{39}$, ce bureau est systématiquement reconstruit par ces derniers. Selon eux, le bureau du droit au logement est désormais « le centre institutionnel et symbolique de la lutte, la maison commune mais aussi le reflet concrétisé de la volonté collective des habitants de la vallée, mais en tout état de cause un lieu qui va au-delà de la signification d'une quelconque association ou coopérative $40 »$.

«Le bureau est un toit qui réunit le quartier et qui nous unit. Les gens qui travaillent au bureau sont très importants pour nous. Ils $\mathrm{y}$ sont toujours restés, parfois sans nourriture, sans eau. Même si tous les habitants du quartier ne viennent pas souvent, nous savons que le bureau reste toujours ouvert et les affaires continuent. Les femmes peuvent y venir confortablement. Ils réussissent à garder la porte du bureau toujours ouverte. Il n'y a jamais de vacances pour le bureau, ni l'été ni l'hiver ${ }^{41}$ ».

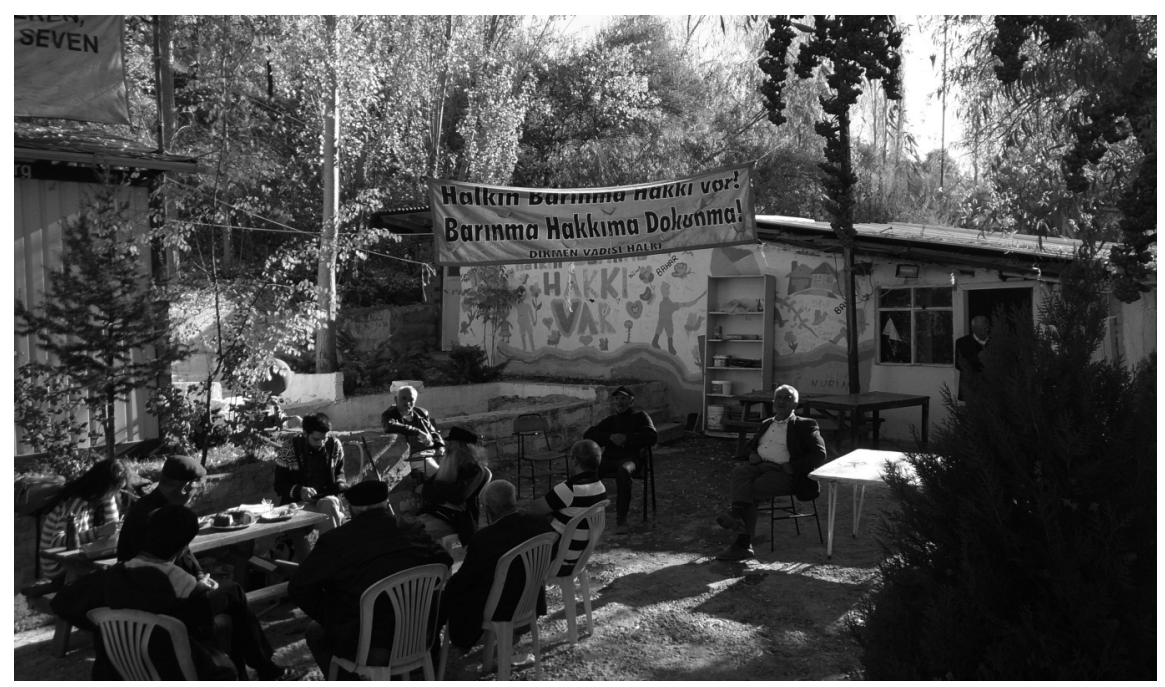

Bureau du Droit au Logement (cliché de l'auteure)

38. Entretiens menés dans le quartier et Özer M., op. cit.

39. Il s'agit d'une accusation relayée par les médias mais qui n'a pas été prouvée jusqu'à l'heure actuelle.

On peut voir http://www.emo.org.tr/genel/bizden_detay.php?kod=62454\&tipi=\&sube=14\#.Vpz8EVKj $\mathrm{Hzg}$

40. Özer M., op. cit., p. 28.

41. Entretien réalisé avec Sultan, Ankara, 29 octobre 2013. 
Dès sa création, de nombreuses actions y ont été organisées, "décidées lors des assemblées générales et collectivement, en informant toujours l'ensemble des habitants qui ont fait le choix de rester et de résister ${ }^{42} »$. Au début, ces actions sont focalisées sur deux points : 1) s'opposer au projet tout en revendiquant une rénovation du quartier, assortie de la garantie que les anciens habitants pourront bénéficier des nouveaux logements sans endettement prolongé ; 2) dénoncer le mépris, la stigmatisation et la tentative de marginalisation du quartier par la mairie d'Ankara.

Pour faire valoir leur droit, les habitants s'adressent d'abord aux syndicats, aux chambres professionnelles et aux organisations politiques. Ils sollicitent leur soutien et leur expertise dans le domaine juridique et de l'urbanisation. Ils tentent également d'obtenir le soutien de la mairie de Çankaya et de quelques députés. Cependant, cette stratégie est rapidement abandonnée car les membres du bureau considèrent qu'elle néglige le pouvoir propre du peuple et transfère la gestion de la lutte aux élites et aux élus. De ce fait, toutes les actions directes sont organisées collectivement par les habitants eux-mêmes. Ils abandonnent également l'idée d'être représentés par un porte-parole et un leader. Ils continuent toutefois à collaborer avec des «élites ", pour la plupart des juristes, des urbanistes et universitaires, afin d'inventer des projets alternatifs comme la construction d'un amphithéâtre et d'un parc pour enfants. Des étudiants de l'Université technique du Moyen-Orient 43 fréquentent régulièrement le quartier pour organiser des projections de film ou des ateliers pour enfants.

Parmi les principales actions des habitants, on compte des sit-in devant le parlement, des déclarations médiatiques devant la mairie d'Ankara, des marches collectives, des rassemblements dans le parc (Güvenpark) de la place centrale d'Ankara (Kizılay), l'organisation d'un festival annuel et de nombreux procès contre la mairie menés avec l'aide d'un avocat engagé au sein d'Halkevleri ${ }^{44}$ (Maisons du peuple), une organisation politique de gauche largement impliquée dans les activités de résistance au sein du quartier. Ainsi, entre 2006 et 2012, les habitants ont recours à tout un répertoire d'action cou-

42. Extrait d'entretien collectif avec les membres du bureau du droit au logement, 28 octobre 2013, Ankara.

43. Une des meilleures universités turques, localisée à Ankara, connue comme une université « de gauche engagée ».

44. Une organisation fondée par les élites fondatrices de la république en 1932 dans l'objectif de familiariser le peuple avec les principes de la République, de fournir des programmes d'éducation et de culture. Elle a été fermée par le gouvernement du Parti démocrate dans les années 1950 et tout son patrimoine a été transféré au Trésor public. Après le renversement de ce parti et la mise en place d'une nouvelle constitution suite à un coup d'État militaire en 1960, l'organisation a été rétablie en 1963 sous un statut d'association indépendante de l'État. Le deuxième coup d'État en 1980 a, de nouveau, interdit cette organisation marquée à gauche (à la gauche radicale), proche des mouvances socialistes patriotiques des années 1970. Halkevleri a recommencé ses activités à partir de 1987 et a opté pour une organisation par en bas au sein des quartiers, refusant tout approche avant-gardiste. 
rant pour faire valoir leur droit et rendre leur cause publique. Ces actions ne nous informent pas seulement sur les stratégies des habitants, elles révèlent également les imaginaires politiques propres à ce quartier, les représentations associées aux autorités, au pouvoir et au politique ou encore la manière dont les identités se construisent et se reconstruisent dans la mobilisation ${ }^{45}$.

Les actions dans la vallée de Dikmen se durcissent à partir du 1 er février 2007 lorsque des représentants de la mairie se présentent accompagnés de 5300 policiers et de bulldozers pour démolir entièrement le quartier. Cette date marque tous les esprits et constitue à la fois un traumatisme et une victoire pour le collectif :

«Ce jour-là, nous avons lutté contre la police neuf heures durant. Nous avons installé des barricades, jeté des pierres, cassé leur grue, blessé des policiers. Des copains se sont allongés devant les panzers mais nous avons réussi. Ils n’ont pas réussi à détruire une seule maison, ils sont partis comme ils sont venus. Certains d'entre nous ont été placés en garde à vue. Le procès continue encore 46 ».

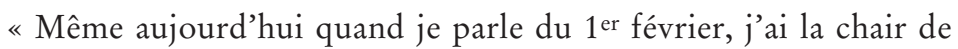
poule, les genoux qui tremblent! Nous avions reçu un papier de la municipalité accordant un délai d'un mois pour vider les maisons. Personne dans le quartier y compris les chiens n'a dormi cette nuit du 30 janvier. Il y avait des milliers de policiers, des panzers, des bulldozers, des grues... comme si c'était un champ de bataille. En plein milieu de Çankaya, c'était comme en Palestine. Froid, hiver, neige... Nous courions d'un côté à l'autre. Tout le monde a pris un bâton, une pierre, personne n'avait peur. Ma fille n'a que 17 ans mais elle était aussi dans la rue. Nous ne les avons pas protégés parce que c'étaient nos enfants 47 ».

De nombreuses personnes évoquent également l'impact de la stratégie agressive de la mairie d'Ankara dans la construction de leur solidarité :

«Moi, je remercie Melih Gökçek, que Dieu le bénisse. Il nous a réunis sous le même toit. Chacun défendait sa maison mais en fait, nous défendions une seule et unique maison, notre quartier. C'est comme ça que nous avons fondé le bureau. Il y a des gens de différentes cultures dans le quartier, des Alévis, des Kurdes... Nous ne nous connaissions pas plus que ça. Aussitôt que nous avons commencé la

45. Goirand C., «Mobilisation et répertoires d'action collective en Amérique latine », Revue internationale de politique comparée, vol. 17, $\mathrm{n}^{\circ} 2,2010$, pp. 7-27.

46. Extrait d'entretien avec Tarik, Ankara, 29 octobre 2013.

47. Entretien avec Gülizar dans Özer M., op. cit., p. 140. 
lutte, tout le monde a commencé à se fréquenter, se familiariser. La mobilisation nous a soudés, elle a renforcé nos liens 48 ».

Une femme raconte comment le maire l'a méprisée quand elle a commencé à évoquer la difficulté que lui posait le paiement des crédits proposés pour les logements neufs en raison du chômage de son mari et de la maladie de son enfant :

«J'ai vu pendant ces sept années de lutte comment un maire pouvait se moquer facilement de la pauvreté du peuple. Quand je lui ai expliqué la situation de mon mari, il m’a répondu "puisque c'est ça, il peut aller vendre des citrons au marché”. Comme si en vendant des citrons, vous pouviez acheter une maison 49 ».

Cet épisode incite d'ailleurs les femmes du quartier à organiser une « campagne de citrons ». Munies de filets de citrons, elles organisent plusieurs sit-in devant le Parlement, la mairie et les ministères, médiatisant ainsi leur cause et leurs conditions de vie dans la vallée de Dikmen.

Ce type d'action, qui s'inscrit dans un clair rapport de force avec le pouvoir politique en place, montre également qu' "à travers les modes de présentation de soi et les stratégies de publicisation des demandes, ce sont aussi les identités des groupes mobilisés, la répartition sociale des ressources politiques ainsi que la façon dont ces groupes conçoivent leur position dans leur société et dans leur système politique qui peuvent se faire jour 50 ».

Le discours de stigmatisation des acteurs publics et notamment du maire d'Ankara, qui insulte et méprise constamment les habitants de la vallée en les traitant parfois de «terroristes » ou de «truands » 51, a accentué, avec le temps, la mobilisation collective tout en politisant le reste des habitants sur la base $\mathrm{du}$ sentiment d'indignation. Cette indignation peut être considérée comme un ressort affectif de la participation et comme une sorte de « choc moral 52 » qui parvient à toucher les cœurs et les consciences au point de constituer de bonnes raisons de protester. D’après James Jasper, ce choc moral « résulte

48. Entretien réalisé avec Türkan, Ankara, 30 octobre 2013.

49. Özer M., op. cit., p. 75.

50. Goirand C., op. cit., p. 17.

51. Le maire, dans un de ses discours juste avant la tentative de destruction du 1 er février 2007 disait la chose suivante : « Notre problème dans la dernière étape de Dikmen vient d'un groupe idéologique au sein du quartier. Ils veulent créer des incidents. Ils se préparent pour ça. Ce sont des provocateurs. Il y en a un qui parle comme le leader d'une organisation terroriste. [...] Les citoyens ne devraient pas croire ces provocateurs. Nous ne ferons aucune concession et toutes les maisons seront détruites " (http://www.istegundem.com/haber/Ekran-basinda-Dikmenlileri-kizdirdi/8521, consulté le 10 avril 2015).

52. Jasper J., The Art of Moral Protest. Culture, Biography and Creativity in Social Movements, Chicago, University of Chicago Press, 1997. 
d'un événement inattendu ou d'une modification imprévue, plus ou moins brusque, de l'environnement des individus ; [il] implique une réaction très vive [...] [il] conduit celui qui y est confronté à jauger et juger la manière dont l'ordre présent du monde semble s'écarter des valeurs auxquelles il adhère 53 » et de ce fait suscite un sentiment de colère, « de nécessité d'une réaction immédiate, qui commande un engagement dans l'action, et ce, en l'absence même des facteurs favorables généralement soulignés par les théories de l'action collective 54 ", notamment sur l'incapacité des démunis à passer à l'action. Dans le cas de Dikmen, le choc moral se produit d'abord au moment de l'annonce du projet, puis il est alimenté par le mépris de la mairie d'Ankara et atteint son paroxysme avec la tentative de destruction du 1 er février 2007 qui incite une grande partie des habitants à se lever contre le projet sans qu'il y ait derrière eux une organisation avant-gardiste assurant un quelconque leadership.

Pour dénoncer la marginalité du quartier et le mépris dont il fait l'objet, les habitants inventent également des formes d'action originales, dont la campagne de citrons évoquée ci-dessus. D’après Michel Agier, des fermetures sociales et spatiales, produites par les stigmatisations, peuvent parfois déboucher sur des formes de franchissement que les citadins mettent en ouvre face aux ségrégations. Agier s'oppose ainsi à l'analyse dominante des marges comme lieu d'anomie sociale et met en évidence les ressources des populations, leurs liens sociaux et leur inventivité. Les actions poursuivies et les stratégies de survie quotidiennes des habitants témoignent en effet, dans le cas qui nous occupe, de cette inventivité face au refus du pouvoir municipal de les considérer comme de véritables interlocuteurs et de reconnaître leur droit à occuper une place au sein de la ville.

\section{Le quartier et la mobilisation : les dimensions spatiales de la résistance}

Situer les mobilisations des habitants au sein de leur quartier face aux projets de transformation urbaine nécessite d'examiner de quelle manière le pouvoir et la résistance sont inscrits dans l'espace. Nous suivons en cela Choukri Hmed pour lequel il est nécessaire de « réintroduire l'espace plus comme une dimension centrale qu'un outil instrumental de l'action collective des groupes démunis ; cette réintroduction ne peut se faire qu'en interrogeant les mécanismes par lesquels les individus et les groupes jouent avec, et se jouent de l'espace et des lieux qu'ils conçoivent, gèrent ou investissent 55 ».

53. Cité dans Traïni C., «Des sentiments aux émotions (et vice-versa). Comment devient-on militant de la cause animale ? ", Revue française de science politique, vol. 60, n² 2, 2010, p. 343.

54. Ibid.

55. Hmed C., «Des mouvements sociaux “sur une tête d'épingle” ? Le rôle de l'espace physique dans le processus contestataire à partir de l'exemple des mobilisations dans les foyers de travailleurs migrants ", Politix, n 84, 2008, p. 148. 
Par rapport à certains quartiers de gecekondu connus dans l'ensemble de la Turquie pour leur engagement politique historique dès les années 1970 et pour la présence en leur sein de militants affiliés à différentes organisations politiques d'extrême gauche, la politisation d'une grande partie des habitants de Dikmen semble être plus strictement liée aux changements spatiaux et à la stigmatisation subis. Le quartier constitue un espace de vie où se tissent des liens, des réseaux et des solidarités sociales et contribue de ce fait, et pour une large part, à la socialisation des individus. Il joue donc un rôle important dans le processus d'identification des individus notamment à travers le sens qu'on lui attribue lors des mobilisations et résistances collectives ${ }^{56}$. De ce fait, toute tentative visant à sa transformation et à sa destruction est considérée par une partie des habitants comme une menace à leur existence au sein de la ville. Cependant, dans la plupart des cas, la peur de se voir éloigner de la ville et de perdre son quartier et les liens sociaux qui le caractérisent ne génère pas systématiquement une résistance, ni nécessairement n'entraîne une politisation des personnes concernées. Bien souvent, les habitants acceptent les projets, les solutions proposées et les décisions prises par les autorités publiques sur leur sort. Dans le cas étudié ici, le rôle mobilisateur du quartier, qui se révèle lors de la résistance et la politisation des habitants qui s'ensuivent, semble être le produit de la menace de disparition du quartier engendrée par le projet et des discours marginalisants de la mairie. Cette combinaison a progressivement éveillé une conscience politique dans le cours de la résistance organisée. Une des habitantes, Sultan, souligne l'importance du quartier : " une maison en dehors de mon quartier ne veut rien dire pour moi. Le quartier a autant de valeur que ma maison. Nous sommes tous des gens pauvres, opprimés. Le quartier est le lieu qui nous réunit tous 57 ». Gülhan, une autre habitante, ajoute que le quartier est une grande et forte famille, le lieu où « les malheurs deviennent du miel 58 ». Dans ce cadre, on observe clairement la réappropriation et la redéfinition du quartier dans le processus de mobilisation et les interactions émergeant parmi les habitants lors de la résistance. Il est très vite qualifié d'indispensable, support de mises en scène et de discours de justification, il est devenu l'espace de référence de la mobilisation ${ }^{59}$. La construction sociale du quartier débouche ainsi sur une politisation progressive de ses habitants.

De nombreuses recherches ont tenté de définir la notion de politisation. Sans entrer dans un vaste débat théorique, et en nous focalisant sur le cas des

56. Voir Neveu, C., « Deux formes de territorialisation de l'engagement dans l'espace urbain », in Bertheleu H., Bourdarias F. (dir.), Les constructions locales du politique [en ligne], Tours, Presses universitaires François-Rabelais, 2008 (généré le 14 janvier 2016). Disponible sur Internet : <http://books.openedition.org/pufr/2434> ; Bonnemaison J., Cambrezy L., Quinty-Bourgeois L., (dir)., Les territoires de l'identité. Le territoire, lien ou frontière ? Tome 1, Paris, L'Harmattan, 1997.

57. Entretien réalisé avec Sultan, Ankara, 10 janvier 2015.

58. Özer M., op. cit., p. 67.

59. Bonny Y., Ollitrault S., Keerle R., Le Caro Y. (dir), Espaces de vie, espaces enjenx. Entre investissements ordinaires et mobilisations politiques, Rennes, PUR, 2011. 
habitants de la vallée de Dikmen, nous nous intéressons particulièrement à la politisation des « individus ordinaires » (par opposition aux professionnels du politique) dans leur vie quotidienne. Pour la politisation, Jacques Lagroye propose une définition substantielle : « la politisation est une requalification des activités sociales les plus diverses, requalification qui résulte d'un accord pratique entre des agents sociaux enclins, pour de multiples raisons, à transgresser ou à remettre en cause la différenciation des espaces d'activité 60 ». Cette définition se limite néanmoins, d'après Camille Hamidi, aux activités au sein des organisations politiques spécialisées compte tenu des exemples qu'il propose ${ }^{61}$. Les définitions ayant tendance à se concentrer sur des activités professionnelles du politique (comme le militantisme au sein d'un parti politique) n'éclairent pas suffisamment sur la nature non institutionnelle, les formes et les causes de la politisation des individus ordinaires. Notre objectif est précisément d'amener l'analyse au-delà de la vision institutionnelle de la politisation ${ }^{62}$. La définition donnée par Hamidi nous semble pertinente dans ce sens pour comprendre la politisation observée en dehors de sphères politiques institutionnalisées. Elle précise deux éléments : la référence aux principes généraux (la montée en généralité) et la reconnaissance de la dimension conflictuelle ${ }^{63}$. Ce qui nous intéresse particulièrement, et qui caractérise la politisation des habitants de Dikmen, ce sont les pratiques et les discours situés en deçà des frontières de la sphère institutionnelle et qui relèvent des «arts de résistance 64 » ou des « tactiques de subalternes 65 ». Considérées dans leur quotidienneté, « [1]es pratiques de résistance subversive et les actes de transgression sous différentes formes [...] des habitants de Dikmen sont analysées et réhabilitées comme des formes de politisation contestataire des individus ordinaires $66 »$. Ces pratiques s'inscrivent non seulement dans une conflictualité avec les acteurs politiques concernés, en l'occurrence la mairie, mais également se réfèrent à un principe général comme « droit au logement pour tous » à partir de leur cas singulier. L'accent est mis sur l'invocation de principes de justice et de la dignité pour le relier au droit au logement ${ }^{67}$.

Avant le lancement du projet, la diversité ethnique, religieuse et politique du quartier avait pour corollaire l'absence d'actions politiques organisées ou de conscience politique susceptible de générer une solidarité collective. Le

60. Lagroye J. (dir.), La Politisation, Paris, Belin, 2003, p. 361.

61. Hamidi C., "Eléments pour une approche interactionniste de la politisation. Engagement associatif et rapport au politique dans des associations locales issues de l'immigration ", Revue française de science politique, vol. 56, $\mathrm{n}^{\circ} 1,2005$, p. 9.

62. Aït-Aoudia M., Bennani-Chraibi M., Contamin J. G., « Indicateurs et vecteurs de la politisation des individus : les vertus heuristiques du croisement des regards ", Critique internationale, $\mathrm{n}^{\circ}$ 50, 2011, p. 9-20.

63. Hamidi C., op. cit, p. 10.

64. De Certeau M., L'Invention du quotidien. 1. Arts de faire, Paris, Gallimard, 1990.

65. Scott J., La Domination et les arts de la résistance, Paris, Éditions Amsterdam, 2008.

66. Ait-Aoudia M., Bennani-Chraibi M., Contamin J. G., art. cit., p. 14.

67. Pitkin C., "Justice : on Relating Public and Private ", Political Theory, vol. 9, n 3, 1981, p. 327-352. 
quartier donnait une image paisible. Il paraissait sans problème manifeste, sans conflit notable entre les habitants d'autant que les différentes parties du quartier n'étaient pas quotidiennement en interaction. Certaines personnes interrogées sur ce point soulignent par exemple la tendance au regroupement des familles originaires d'une même ville anatolienne qui avaient élu domicile dans un coin bien précis du quartier. Des échanges quotidiens liés à la fréquentation de l'épicier, de l'arrêt de bus ou du café de quartier existaient, mais en l'absence d'un risque commun de destruction de leurs maisons ou de leurs lieux de vie, ces échanges restaient limités. Chacun préférait vivre ses sociabilités dans sa rue, avec ses voisins, les gens qu'il connaissait de par sa famille ou sa ville d'origine ${ }^{68}$. La vallée de Dikmen était, en somme, un quartier d'Ankara sans histoires, sans incidents. Le projet de transformation urbaine a entraîné non seulement le développement d'une conscience politique au sein du quartier, mais également le renforcement de la signification du quartier comme un espace de subsistance, un espace-enjeu 69. Dans un contexte d'instabilité spatiale ${ }^{70}$, les résidents ont en effet démontré aux autres et à euxmêmes l'existence d'un potentiel protestataire réel, et la possibilité d'actions collectives.

L'analyse de cette situation particulière requiert de s'interroger à la fois sur le sens octroyé à l'espace par les acteurs des mobilisations, et sur la manière dont ils affirment leur existence au sein d'un espace - le quartier de la vallée de Dikmen -, qui leur donne tout leur sens. Autrement dit, nous tentons ici de comprendre les interactions entre l'espace et la résistance, en lien avec les processus d'identification par lesquels les participants découvrent, transforment, redéfinissent leur relation avec l'espace concerné. Sur la base des entretiens menés au sein du quartier, nous pouvons avancer que l'attachement au lieu et la signification qu'il revêt dans l'identification des habitants sont particulièrement marqués. Le quartier et la ville suscitent ici un fort sentiment d'appartenance, et la résistance vise avant tout à préserver le lieu et les pratiques sociales et culturelles de la vie quotidienne qui s'y rattachent. Dans le cas qui nous occupe, cette résistance s'exprime par une proclamation des habitants qui rappellent ainsi que ce territoire est le leur et qu'il est le support d'une identité collective. Ils revendiquent leur droit à occuper ce quartier, à se l'approprier et à le préserver. L'appropriation de cet espace permet de créer une identité spatiale individuelle, pour reprendre la formule de Michel Lussault ${ }^{71}$, qui permet à son tour aux habitants de se positionner socialement

68. Tissot S., «Entre soi et les autres », Actes de la recherche en sciences sociales, $\mathrm{n}^{\circ} 204,2014$, pp. 4-9.

69. Bonny T., Ollitrault S., Keerle R., Le Caro Y. (dir), Espaces de vie, espaces enjeux. Entre investissements ordinaires et mobilisations politiques, op. cit.

70. Le Texier E., Quand les exclus font de la politique... Le barrio mexicain de San Diego, Californie, Paris, Presses de Sciences Po, 2006, p. 131.

71. Lussault M., "Identité spatiale », in Lévy J., Lussault M. (dir.), Dictionnaire de la géographie et de l'espace des sociétés, Paris, Belin, pp. 480-481. 
et d'expérimenter chacun une image de soi et des autres. Comme le montre Lilian Mathieu dans sa recherche sur les mobilisations des prostituées à Lyon, la ville ou le quartier correspondent ici à des « lieux sacrés où l'action prend une signification renforcée aux yeux des participants aussi bien que des témoins 72 ». Ce qui implique que "certaines actions publiques peuvent être vécues comme des profanations pour ceux qui s'estiment propriétaires d'un territoire ${ }^{73} »$.

Ces visions antagonistes sur la ville nous renvoient à l'idée d'Henri Lefebvre concernant la production de l'espace. Pour lui, l'espace intègre, en soi, différentes interprétations possibles en fonction de la position des acteurs concernés. Les habitants d'une ville en sont ses véritables propriétaires et, de ce fait, revendiquer le droit d'y habiter et de pouvoir accéder à toutes les infrastructures nécessaires pour mener une vie décente est un droit fondamental que Lefebvre appelle le droit à la ville ${ }^{74}$. La demande des habitants de Dikmen relève clairement d'une revendication d'un droit à la ville, dans la mesure où leur volonté de participer à la conception de leur espace de vie procède d'une volonté de rendre la ville plus inclusive, de voir les opportunités mieux réparties au sein de la population. Ainsi, à travers leurs affrontements avec les acteurs publics, les habitants de la vallée expriment leur désir de participer ouvertement et équitablement aux processus de production de l'espace urbain et d'accéder aux avantages de la vie citadine. Ils font valoir que vivre dans le centre-ville ne doit pas être un luxe et s'opposent à toutes formes de ségrégation et de confinement spatiaux imposés par le haut.

Les habitants exigent d'être reconnus en tant que citoyens à part entière ; ils réclament l'annulation du projet et la conservation des lieux. Ils veulent, en quelque sorte, que les caractéristiques sociales et culturelles de leur quartier soient préservées, même dans l'hypothèse où un projet de rénovation serait mené à bien ; ils souhaitent qu'il demeure "un endroit sûr » à l'intérieur duquel « les occupants bénéficient d'une protection contre l'intervention des autorités et des ennemis 75 ». Il ressort des données recueillies que pour les habitants de Dikmen, le projet de transformation est synonyme de déracinement, de rupture dans leur histoire collective. Dans l'organisation de leur vie quotidienne, ils adoptent de ce fait des stratégies quotidiennes ordinaires que l'on peut considérer comme des résistances invisibles leur permettant de s'approprier l'espace et de consolider les liens d'appartenance au quartier. Au sein du bureau du droit au logement, par exemple, il a été décidé que chaque "propriétaire » de gecekondu devrait faire en sorte que son jardin soit beau, propre et accueillant, que chacun devrait participer au nettoyage du quartier et s'en-

72. L'expression de « lieux sacrés » provient de William H. Sewell, cité dans Mathieu L., op. cit., p. 193. Nous avons préféré utiliser la traduction fournie par L. Mathieu.

73. Idem.

74. Lefebvre H., Le Droit à la ville, op. cit.

75. Mathieu L., op. cit., p. 194. 
gager à ne pas y jeter ses poubelles, notamment dans les bâtiments en ruine. L'installation de chiffonniers et de réfugiés syriens dans les maisons abandonnées, également, a été discutée. Les habitants pensent que ces derniers y ont été incités par le maire d'Ankara dans le but de provoquer un conflit et de créer un climat de mécontentement dans le quartier. Toutefois, afin de renforcer la réputation de tolérance et de convivialité de ce quartier multiethnique et pour déjouer la stratégie du maire, il a été décidé d'accepter ces nouveaux venus et de les inviter aux réunions dans le souci d'établir collectivement des règles $\mathrm{du}$ « vivre ensemble ».

Ces actes peuvent être interprétés comme la combinaison de tactiques et de stratégies subversives qui ne sont pas toujours manifestes mais qui consistent à contester les règles du jeu imposées par les acteurs dominants. La tactique est, selon Michel de Certeau, l' « art du faible », de celui à qui le pouvoir de contrôle fait défaut ${ }^{76}$. Elle consiste à mettre à profit cette faiblesse en identifiant la force de l'autre pour mieux l'exploiter à ses propres fins. «Elle s'infiltre furtivement dans des lieux stratégiques propres, profitant des opportunités momentanées d'action 77 ». On peut donc parler de l'émergence d'une " culture subalterne 78 » spécifique au quartier, visible et compréhensible uniquement en son sein et par ses membres, et qui permet de constituer une résistance invisible.

\section{Les acteurs de la politisation dans le quartier}

Si la politisation des habitants se développe principalement au gré de leur propre expérience, dans le cadre de la lutte contre la mairie, elle ne surgit pas pour autant du néant. Certains paramètres préexistants permettent son émergence. En premier lieu, si le quartier pouvait paraître «dépolitisé » avant le projet d'urbanisation, c'était lié au fait que l'engagement politique y était un acte individuel et non collectif. Autrement dit, le quartier comptait des personnes, notamment parmi ses fondateurs, qui étaient membres d'Halkevleri et qui avaient participé aux mobilisations d'extrême gauche dans les années 1970. Fortes de leur capital militant historique, celles-ci vont jouer un rôle décisif dans la mise en place de la mobilisation et l'organisation de la résistance autour du bureau du droit au logement. En effet, leur investissement militant attire l'attention de leur organisation sur le quartier de la vallée de Dikmen. Cette organisation et ses militants mèneront, dès lors, une lutte quotidienne constante non seulement en termes de protection physique du quartier (par exemple en participant aux barricades lors des tentatives de destruction) mais également en partageant leur savoir-faire. Ces militants, que l'on peut qualifier de passeurs d'action, ont assuré une capacité d'expertise aux mobilisations.

76. De Certeau M., op. cit.

77. Dorfman E., "Michel de Certeau et l'écriture du quotidien », Tétrade, n 1, 2014, p. 73.

78. Scott J., op. cit. 
En second lieu, la mobilisation est également facilitée par la présence de la communauté alévie dans le quartier. De par leur passé, ils ont acquis une culture de la résistance et une primo-socialisation politique qui sert depuis lors de base à tout type de mobilisation et d'action collective. Les toutes premières réunions contre le projet de transformation ont d'ailleurs été réalisées dans le Cemevi (lieu de culte des Alévis) malgré la réticence de certains habitants.

\section{L'investissement des femmes par l'appropriation du quartier}

Une autre originalité de la résistance à Dikmen est la participation active des femmes dans la mobilisation. On peut même dire que c'est grâce à elles que le quartier demeure mobilisé depuis tant d'années car elles entretiennent à son égard un rapport particulier, différent de celui des hommes. La majorité des hommes sont absents dans la journée et ne rentrent chez eux que le soir. Ils ont également une vie et des réseaux amicaux à l'extérieur du quartier tandis que la plupart des femmes y sont en permanence, fabriquent et développent leurs liens sociaux en son sein. Elles interviennent à plusieurs titres dans la vie quotidienne du quartier qu'elles perçoivent comme structurant leur vie. Il constitue le lieu où les manques sont compensés par la solidarité, où les familles et proches se retrouvent et où l'on a le sentiment d'être sur son propre territoire. De ce fait, il donne les moyens d'exister dans la ville et de s'y accrocher :

«Avant cette lutte, je ne connaissais pas beaucoup de monde dans le quartier. La lutte nous a réunies. Sans faire de distinctions religieuse, linguistique ou ethnique, nous sommes devenues comme des sœurs pour partager nos malheurs, nos bonheurs, nos espoirs. Nous, les femmes, avons appris que la vie n'est pas que dans nos maisons mais aussi dans les barricades. Alors que nous ne sortions pratiquement jamais et que nous finissions la journée entre quatre murs, nous sommes aujourd'hui partout avec nos enfants. Nous avons appris à réclamer nos droits et nous l'apprenons à nos enfants pour qu'ils ne vivent pas la même chose. Nous avons appris à réclamer la vie que nous désirons et non celle que l'on nous impose. La vallée est devenue une grande école d'apprentissage pour les femmes ${ }^{79}{ }$.

La plupart de femmes n'exercent pas de métier rémunéré. Quelques-unes cependant travaillent comme femmes de ménage dans les quartiers de luxe qui entourent la vallée. Elles ne développent donc aucune autre forme de relations sociales en dehors de leur quartier, si ce n'est, pour les secondes, des relations de travail avec les patronnes qui les emploient. Le quartier est donc l'unique lieu où elles s'accomplissent, nouent leurs amitiés, passent leur temps et investissent l'espace (échanges de voisinage, bavardages quotidiens et séances de thé 
en fin de journée dans les jardins, déplacements chez l'épicier, surveillance des enfants dans la rue, etc.).

C'est le risque de voir disparaître leur quartier et leurs réseaux sociaux de solidarité qui motive en premier lieu le passage des femmes à l'action. Leur attachement à ce lieu se traduit par un sentiment de bien-être ; a contrario, devoir le quitter provoque un sentiment de perte ${ }^{80}$. L'analyse des propos recueillis et des comportements observés permet de mettre en exergue la signification politique des pratiques et des usages du quartier ${ }^{81}$ et de confirmer que l'espace joue un rôle déterminant dans le passage à l'action. Il motive et structure la résistance des femmes et transforme de ce fait les relations en son sein. Les femmes se trouvent le plus souvent à l'origine même des principaux liens sociaux du quartier. Le genre apparaît ici comme une variable-clé favorisant la participation politique. En somme, la défense du quartier comme source de politisation des femmes les transforme en leur conférant une confiance en elles, une émancipation individuelle et une reconnaissance collective malgré les obstacles qu'elles ont rencontrés au début. Une femme nous a expliqué par exemple comment son mari l'a tout d'abord empêchée de participer à la manifestation et comment ensuite, devant sa détermination, il l'a finalement autorisée à sortir pour participer à la lutte collective :

«Certains m’ont demandé pourquoi je me prenais la tête avec tout ça mais j'ai continué parce que j'étais persuadée que notre lutte était juste. Je n'ai pas pu aller à certaines mobilisations. Mon mari ne voulait pas. On n'était pas du tout d'accord. On se disputait sans arrêt. Pour moi, c'était injuste. Il n'était pas correct pour moi de rester à la maison alors que des gens luttaient contre le projet. Au bout d'un certain temps, j'ai pu convaincre mon mari et face à mon entêtement, il a accepté la situation. Il n'avait pas le choix, il a vu à quel point j'étais déterminée 82 ».

En octroyant une visibilité sociale et politique à ces femmes souvent invisibles et ignorées dans la ville, en dehors de leur quartier, cette mobilisation affecte également l'organisation spatiale et les rapports de force entre les hommes et les femmes à l'intérieur du quartier.

Les femmes de la vallée découvrent des espaces urbains auxquels elles n'avaient jamais eu accès auparavant en participant aux actions, conférences, panels et pique-niques. Dans le même temps, elles se redécouvrent elles-

80. Guérin-Pacé F., «Le quartier entre appartenance et attachement : une échelle identitaire ? ", in Bacqué M.-H., Guérin-Pace F., Authier J. Y. (dir.), Le Quartier, Paris, La Découverte, 2007, pp. 151-162.

81. Le Texier E., op. cit., p. 113.

82. Les propos de Nur pendant l'entretien collectif avec des femmes de la Vallée de Dikmen, 29 octobre 2013. 
mêmes et mesurent leur capacité réelle à agir. De nouveaux espaces publics sont ainsi identifiés par et pour les femmes au-delà des frontières du quartier. Se rendre au centre-ville pour assister à un panel, y écouter des psychologues, des avocats, des journalistes ou des urbanistes leur permet d'accéder à un savoir inconnu auparavant et d'acquérir de nouveaux savoir-faire favorisant leur émancipation et leur épanouissement personnel et collectif.

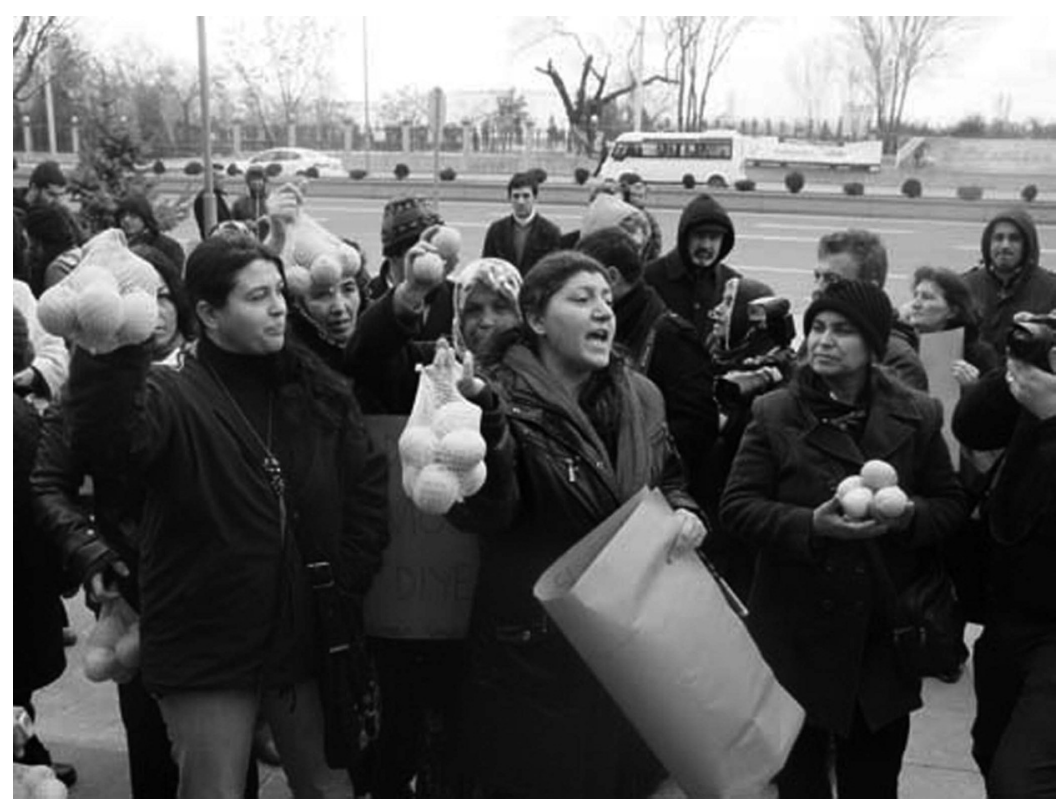

Femmes de Dikmen

Par ailleurs, les besoins pratiques et quotidiens des femmes se transforment en des besoins stratégiques comme la défense de leur maison. Ce processus assure à leurs stratégies de survie et de résistance un sens politique. Le fait qu'elles perçoivent le quartier comme un espace public commun constitué des réseaux informels de communication et de solidarité les conduit à une ouverture sur la ville et leur permet de s'affirmer dans les espaces publics. Leur politisation entraîne une montée en généralité de leur action. Elles commencent à participer à d'autres mobilisations sans lien avec le droit au logement. Elles soutiennent par exemple les mobilisations pour le Gezi Park et la grève des ouvriers de Tekel ${ }^{83}$. Leur revendication d'un droit au logement évolue vers d'autres revendications d'autres droits. Sultan explique ce processus de politisation généralisée :

83. Ce mouvement a été lancé par les ouvriers contre la privatisation de la branche « tabac » de l'entreprise publique TEKEL (Monopole gérant la production et la vente du tabac et de l'alcool en Turquie) et des licenciements massifs. Le mouvement a débuté en décembre 2009 à Ankara et duré pendant des mois résultant sur une grève générale suivie dans différentes villes. Il est considéré un des mouvements sociaux les plus importants des deux dernières décennies en Turquie. 
"S’il n’y a pas de femmes lors d'une mobilisation, elle est vouée à l'échec. Nous avons commencé, au départ, avec le droit au logement mais nous avons compris que l'on ne peut pas politiser la lutte locale de notre quartier, les habitants peuvent facilement l'oublier après avoir récupéré un logement. [...] Pour moi, la résistance, c’est pour la vie. Après la résistance dans le quartier, les femmes de Dikmen ont commencé à manifester partout. Nous sommes allées à la fête du travail, aux mobilisations pour le droit à l'éducation, le droit aux services publics. Pour nous, les droits humains sont indivisibles. ${ }^{84}$ »

Cependant, cette implication active des femmes ne se déroule pas sans critiques. D'après leur dire, certaines d'entre elles sont vivement fustigées par les membres de leur famille :

«Dans ma famille, tout le monde soutient l'AKP. Ils me disent toujours que l'on se bat pour rien, que l'on ne s'oppose pas à l'État, qu'il nous écrasera. Ils m'accusent de me joindre aux contestataires, de leur ressembler. Ils disent “qu'est-ce que l'AKP fait de mal ? Ils construisent nos routes, nos hôpitaux, ils nous donnent des aides sociales”. Ils nous accusent d'être ingrats. Ils m'ont dit : “toi aussi tu es devenue gauchiste, tu votes comme eux, tu es devenue une Alévi” 85 ».

Un des résultats emblématiques de cette politisation a été la désignation d'une femme lors des élections municipales de mars 2014. Candidate au poste de mukhtar 86 auquel les habitants de la Vallée sont rattachés ${ }^{87}$, elle a mené une campagne à l'échelle locale et obtenu 487 voix sur 1200 sans remporter les élections.

Ainsi, cette expérience semble prouver que les asymétries de genre, la division du travail et les différentiels de pouvoir ne doivent pas seulement être conçus comme des contraintes orientant le militantisme des femmes mais également comme des catalyseurs potentiels du passage à l'action ${ }^{88}$. La situation a priori subalterne des femmes du quartier leur a permis de se réunir, de discuter en commun de leur problème collectif et de passer à l'action.

84. Entretien collectif avec les femmes du quartier, Ankara, 12 janvier 2015.

85. Les propos de Fatma pendant l'entretien collectif avec des femmes de la Vallée de Dikmen, 29 octobre 2013.

86. Responsable du quartier administratif correspondant à la plus petite unité administrative en Turquie. Le quartier entendu ici n'a pas le même sens que nous utilisons dans cet article. Il se réfère à un découpage administratif, bien défini cette fois-ci.

87. Le mukhtar (muhtar) est élu pour chaque quartier et s'occupe des affaires de l'état civil et du registre des domiciles des habitants. Échelon le plus bas de la représentation administrative, il est élu au suffrage universel direct mais est considéré comme un fonctionnaire de l'État.

88. Neuhouser K., "Worse than men": An analysis of gendered mobilization in an urban Brazilian squatter settlement, 1971-91 », Gender E Society, vol. 9, n 1, 1995, p. 38-58. 
Nous avons évoqué au début de cette section la présence des militants professionnels aux côtés des habitants non pas pour les "guider » mais pour les accompagner dans leurs diverses résistances. Ces acteurs se distinguent par leur aptitude à intervenir dans différentes arènes dont « les règles, les procédures, les savoirs et les représentations peuvent être sensiblement éloignés. Ils sont tenus de maîtriser une pluralité de rôles et de connaissances, et de les mettre en œuvre en différents lieux de l'espace social 89 ». Dans le cas exemplaire étudié ici, on observe deux catégories de passeurs d'action. Ceux qui sont extérieurs au quartier mais qui accompagnent les habitants à chaque fois qu'il faut mener une action, une campagne ou affronter un procès. Ils permettent à la mobilisation d'explorer de nouveaux terrains ou de nouveaux outils comme le recours au droit par exemple. Ils offrent de nouveaux moyens et compétences aux habitants « ordinaires». Grâce à leur capacité de contre-expertise, « les habitants finissent par posséder un savoir sur ces choses et deviennent tout aussi experts que celui des experts en titre 90 ». Ils développent ainsi leurs capacités d'user de stratégies de survie face à différents interlocuteurs publics. En décembre 2014, par exemple, à la suite d'une information reçue selon laquelle la municipalité s'apprêtait à vendre l'ensemble des terrains du quartier, les habitants se sont organisés pour se porter partie civile et empêcher l'organisation de l'offre publique en faisant valoir son caractère illégal ${ }^{91}$.

La seconde catégorie de "passeurs d'action » est composée des militants de Halkevleri, auparavant étrangers au quartier, mais qui ont fait le choix de s'y installer et d'y vivre pour participer à la contestation. Ces militants sont, en général, masculins et relativement jeunes (entre 25 et 35 ans), sans attache familiale, souvent étudiants à l'université, à moins qu'ils aient arrêté leurs études à un moment donné. Ils adoptent les stratégies des organisations de gauche des années 1970 fondées sur l'idée que le succès de la défense d'une cause politique dépend de l'interaction quotidienne des militants avec le peuple, ce qui implique de vivre dans des quartiers populaires et de travailler dans des usines pour expérimenter la condition ouvrière. Leur rôle est de maintenir les habitants mobilisés et politisés et d'élargir leur conscience politique à l'ensemble des questions de société.

89. Nay O., Smith A., "Les intermédiaires en politique : médiations et jeux d'institutions », in Nay O. et Smith A. (dir.), Le Gouvernement du compromis. Courtiers et généralistes dans l'action politique, Paris, Economica, 2002, p. 12 (http://ceraps.univlille2.fr/fileadmin/user_upload/enseignants/Nay/NAY__SMITH Les_intermediaires_en_politique.pdf, consulté le 27 avril 2015).

90. Boullier D., "Choses du public et choses du politique. Pour une anthropologie des inouïs », in Carrel M., Ion J., Neveu C. (dir.), Les Intermittences de la démocratie. Formes d'action et visibilité citoyenne dans la ville, Paris, L'Harmattan, 2009, p. 29.

91. Les informations et les images liées à cette action peuvent être consultées sur les sites suivants : www.hurriyet.com.tr/ankara/27755090.asp et http://capul.tv/dikmen-vadisi-halkigokcekin-ihalesine-girmeye-calisti-polis-vadililere-saldirdi-7-gozalti/ (consultés le 7 mai 2015). 
Au sein du quartier, ces passeurs se démarquent par leur investissement spatial. Cinq ou six militants de Halkevleri non originaires du lieu s'y sont installés il y a quelques années dans le but de faire vivre l'esprit contestataire et d'apporter leur savoir-faire dans l'action. Ils expliquent ce choix de la manière suivante : "Nous avons voulu sortir du cadre classique d'affrontement dans la rue. Au lieu d'entrer en conflit avec la police dans des émeutes, nous avons préféré consacrer notre énergie à construire une vie dans la Vallée ${ }^{92}$ ».

D’après l'un de ces militants, ils considèrent qu'ils ont réussi leur mission de politisation progressive et d'éveil de la conscience des habitants du quartier :

"Même si au départ il n’y avait pas beaucoup de monde, nous avons mis en place des soirées de réunion informelles. Les gens venaient, parlaient des choses qui ne leur plaisaient pas. Nous avons pensé que s'il faut prendre des risques, il faut les prendre avec les habitants. Il faut faire ce qu'ils ont envie de faire. Ils ont voulu aller à l'AKP en disant qu'ils connaissaient des gens. Ils ont voulu aller à la préfecture, à la police, à la mairie. Nous n'avons jamais donné notre propre avis sur ces institutions. À force de voir qu'on leur fermait la porte au nez, ils ont vu que la seule force qu'ils avaient était euxmêmes. Ils ont dit : si nous résistons dans le quartier, nous pouvons sauver notre maison, défendre notre avenir 93 ».

Ces militants professionnels sont acceptés par les habitants qui se sont convaincus progressivement de leur sincérité. Ils sont collectivement logés gratuitement dans un des gecekondus du quartier qui est devenu un lieu de rencontre pour les jeunes, d'organisation de soirées de poésie et/ou d'aide scolaire pour les collégiens. Dans ce cas-là, l'enjeu est moins leur capacité à imposer un point de vue définitif que leur aptitude à mobiliser. Ils communiquent constamment avec les habitants pour produire des arguments, formuler une représentation commune de la situation. Ils participent et contribuent, de ce fait, à la création d'un « nous », et à l'émergence d'un sentiment de communauté de destin.

92. Entretien réalisé avec Serkan, militant de Halkevleri, Ankara-Kızılay, 15 novembre 2013.

93. Entretien réalisé avec Özgür, militant de Halkevleri, Ankara-Kızılay, 15 novembre 2013. 
À la lumière de cette étude de la mobilisation de la vallée de Dikmen, un constat s'impose : les lieux sont investis de sens et ils ménagent en tant que tels un champ d'action. L'espace apparaît comme un élément déterminant du passage à l'action. Dans cette perspective, il peut être appréhendé comme un opérateur de relations sociales, non seulement dans sa dimension proprement physique mais également symbolique, ainsi que dans une « relation double de potentialité et de contrainte » façonnant les mobilisations ${ }^{94}$. C'est dans l'espace que viennent se loger toutes les représentations partagées par les individus, et c'est en même temps lui qui détermine leur agencement aussi bien physique que mental. Les lieux sont donc tout à la fois le terrain et l'enjeu des politiques de la contestation. Les actions collectives s'inscrivent dans des lieux physiques, qui soit ont déjà une signification particulière ${ }^{95}$ soit font l'objet d'une construction de sens pendant la mobilisation. Le cas du quartier de la vallée de Dikmen s'inscrit dans une tendance à la montée généralisée des contestations urbaines ces derniers années, que ce soit en Espagne, en Grèce, en Pologne ou ailleurs ${ }^{96}$, qui laisse à penser que la ville, et particulièrement le quartier, constitueraient le nouvel espace d'expression des revendications identitaires et politiques. Comme l'explique le sociologue Denis Merklen, «le politique se délocalise vers les quartiers dès lors que les classes populaires se mobilisent pour que leur lieu d'habitation ne soit pas exclu de l'espace public, un lieu d'habitation qui est, par ailleurs, massivement visé par l'action de l'État. [...] Cette localisation du politique a profondément changé la "politicité" populaire. Le quartier se constitue ainsi comme territoire de l'action et du déploiement de formes plus ou moins nouvelles de mobilisation, individuelle et collectives $97 »$. Des quartiers pendant longtemps marginalisés par diverses politiques publiques d'aménagement du territoire, d'accès aux services publics et de distribution de prestations sociales n'attirent l'attention que lorsque leurs habitants décident de passer à l'action, le mettant alors au centre des débats sur la distribution des richesses, la cohésion sociale, les inégalités socio-spatiales et la participation politique.

Nous retiendrons que la résistance des habitants du quartier de la vallée de Dikmen peut être analysée comme une forme de lutte pour la reconnaissance dans la mesure où ils affirment ainsi leur droit d'exister dans la ville, de se l'approprier et de l'investir avec leurs propres modes de vie et façons d'être. Cette affirmation ne passe pas systématiquement par des mobilisations visibles mais aussi par le déploiement de tactiques de survie au quotidien et de

94. Auyero J., «L'espace des luttes. Topographie des mobilisations collectives », Actes de la recherche en sciences sociales, $\mathrm{n}^{\circ} 160,2005, \mathrm{p} .126$.

95. Ibid., p. 130.

96. Voir Jacobsson K. (dir.), Urban Grassroots Movements in Central and Eastern Europe, Londres, Ashgate, 2015.

97. Merklen D., op. cit., p. 266. 
réseaux informels de solidarité, ainsi que par la transgression des règles imposées par les acteurs dominants. Notons enfin que les élections législatives de juin 2015 ont dissuadé la mairie de durcir le ton envers les habitants de ce quartier qui restent encore très mobilisés. Au moment de la rédaction de cet article (janvier 2016), les habitants sont, semble-t-il, sur le point de signer un accord de principe avec le maire dans l'objectif de récupérer des logements sur place au sein du projet, écartant de ce fait le risque d'un déplacement forcé et de perte de leur vie sociale au sein de leur quartier. 